\title{
President's Corner (Mitteilungen der Deutschen Schmerzgesellschaft e.V.)
}

Schmerz 2021 · 35:223-225

https://doi.org/10.1007/s00482-02100555-4

(c) Springer Medizin Verlag $\mathrm{GmbH}$, ein Teil von Springer Nature 2021

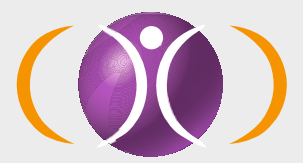

Deutsche Schmerzgesellschaft e.V. Sektion der International Association for the Study of Pain (IASP)
Mitteilungen der Deutschen Schmerzgesellschaft e.V.

Bundesgeschäftsstelle

Alt-Moabit 101 b, 10559 Berlin

Tel. 030/39409689-0 Fax 030/39409689-9

info@Schmerzgesellschaft.de www.schmerzgesellschaft.de

Geschäftsführer

Thomas Isenberg, presse@Schmerzgesellschaft.de (V.i.S.d.P.)
Liebe Mitglieder der Deutschen Schmerzgesellschaft e. V., liebe Kolleginnen und Kollegen!

Zugegeben: Nach über einem Jahr Pandemie und Bekämpfung von COVID-19 ersehnen wir eine erhebliche Steigerung der Impfquoten, hoffen auf wenig relevante Mutationen des $\mathrm{Vi}$ rus und Sonnenstrahlen für das Gemüt im Sommer. Zum Thema COVID und Schmerz möchte ich Ihnen über ein Teilprojekt des „Netzwerks Universitätsmedizin“ (NUM) berichten. In diesem Rahmen werden derzeit 13 Projekte mit dem Ziel gefördert, Maßnahmenpläne, Diagnostik- und Behandlungsstrategien möglichst aller deutschen Universitätskliniken zusammenzuführen und auszuwerten. In einem der Projekte ist auch die Schmerzmedizin vertreten: Das Projekt „Nationales Pandemie Kohorten Netz" (NAPKON) https://napkon.de/ baut unter anderem die Infrastruktur für mehrere Kohorten und Biobanken auf, an denen Krankheitsverläufe standardisiert beobachtet und Patienten nachbefragt werden können, teilweise auch über längere Zeiträume. Dazu wurden fach- und organspezifische Arbeitsgruppen (FOSA) eingerichtet, unter anderem auch eine FOSA Schmerzmedizin. Prof. Winfried Häuser koordiniert diese Arbeitsgruppe, in der eine Reihe von weiteren Mitgliedern unserer Gesellschaft mitwirken, um schmerzspezifische Aspekte und insbesondere relevante Befragungsinstrumente dort $\mathrm{zu}$ implementieren. Vielen Dank an dieser Stelle!
Spannende forschungsorientierte Neuigkeiten verkündet auch die IASP: Diese hat kürzlich eine Erklärung zur Verwendung von Cannabinoiden bei Schmerzen herausgegeben. https://www. iasp-pain.org/PublicationsNews/ NewsDetail.aspx?ItemNumber= $11145 \&$ navItem Number $=643$. Grundlage ist ein detailliertes systematisches Review, an dem eine Task Force der IASP, geleitet durch Prof. Andrew Rice, 2 $1 / 2$ Jahre lang gearbeitet hat. $\mathrm{Zu}-$ sätzlich zu der Erklärung sind in der Zeitschrift PAIN eine Reihe von 13 wissenschaftlichen Artikeln veröffentlicht worden, die die Evidenz zu diesem Thema umfassend darstellen. Zusammenfassend kann die IASP aufgrund fehlender Belege aus qualitativ hochwertiger Forschung die allgemeine Verwendung von Cannabinoiden in der Schmerzbehandlung aktuell nicht befürworten. Dahingegen soll die Erfahrung von Menschen, die bezüglich ihrer Schmerzen von Cannabinoiden profitiert haben, nicht geringgeschätzt werden. Die IASP fordert eine Forschungsagenda, um Wirkung und unerwünschte Wirkungen im Zusammenhang mit dem Einsatz von medizinischem Cannabis, Arzneimitteln auf Cannabisbasis und synthetischen Cannabinoiden besser zu verstehen.

Erfreulich haben sich auch die Aktivitäten zur Stärkung unserer Nachwuchsarbeit entwickelt:

Am 19. und 20. März 2021 fand die erste WinterSchool der Deutschen Schmerzgesellschaft e. V. unter Federführung des Arbeitskreises Junge Schmerzgesellschaft statt (- Abb. 1).

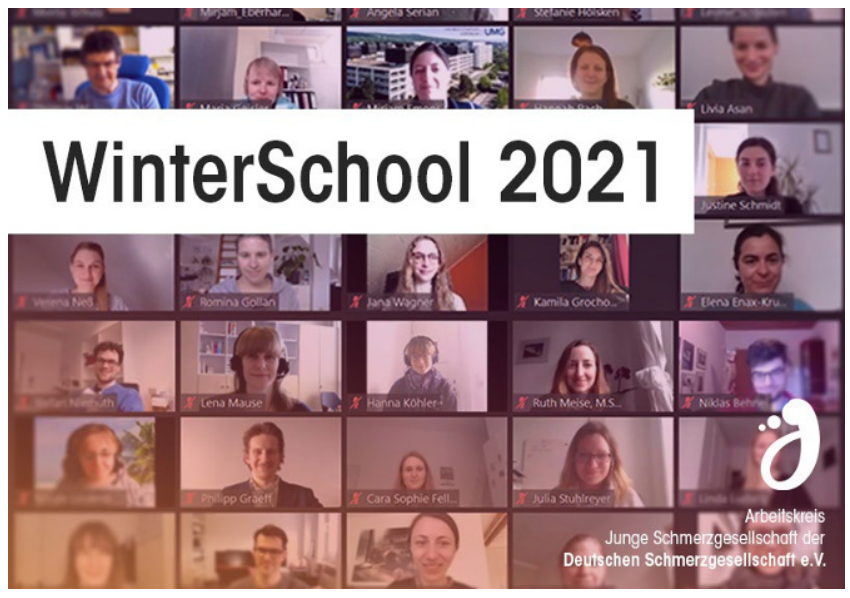

Abb. $1 \Delta$ Winterschool des AK Junge Schmerzgesellschaft

In interdisziplinären Vorträgen von ausgewählten Expertinnen und Experten bzw. und Jungforscher/-innen erhielten die Teilnehmenden die Chance, das wissenschaftliche Feld der Schmerzmedizin zu erkunden und grundlegende wissenschaftliche Fähigkeiten zu erlernen, zum Beispiel in Statistik. Die Stimmen des Feed-Backs (z.B. „Durch die WinterSchool habe ich für meine Forschungsarbeit viele Anregungen, neue Ideen und erweiterte Fragestellungen erhalten, was mich für meine weitere Forschung sehr motiviert!", Frau D., MedizinStudierende und Doktorandin an der Universität Duisburg-Essen, oder aber Frau M, Physiotherapeutin (M. Sc.) in Bochum: „Die WinterSchool ist eine ideale Plattform zur Vernetzung junger Schmerzforscher/-innen aller beteiligten Disziplinen.") sowie deren Detailevaluationsbögen zeigen: Ein super Auftakt - und beim nächsten Mal dann hoffentlich nicht ausschließlich online sondern in einem persönlichen Treffen. Herzlichen Dank an alle Aktiven in unserer Gesellschaft. Wir bleiben gemeinsam gut auf Kurs!

Schließlich möchte ich Sie noch aufmerksam machen auf unser traditionelles Drei-Länder-Treffen, das auch in diesem Jahr leider nicht vor Ort in der schönen Schweiz, aber zumindest virtuell, stattfindet, und ein spannendes Programm verspricht: www.achd-tagung.com (• Abb. 2).

Bleiben Sie gesund! Herzliche Grüße, Ihr

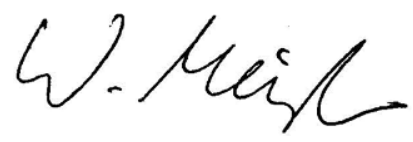

Apl. Prof. Dr. Winfried Meißner Präsident Deutsche Schmerzgesellschaft e.V. 


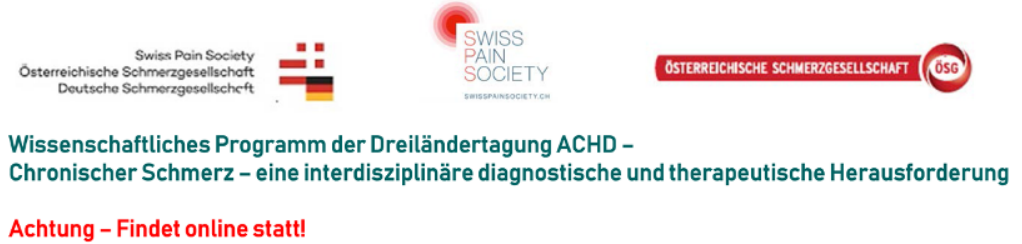

Achtung - Findet online statt!

Freitag, 23. April 2021

$12.30-13,00$

Eröffnung, Grussworte

PD Dr. Konrad Maurer. SPS

PD Prim. Dr. Nenad Mitrovic, ÖSG

Prof. Dr. Winfried Meissner, DSG

Dr. med. André Ljutow, Chefarzt, Zentrum für Schmerzmedizin Nottwil

$13.00-15.00$

1. Sitzung: Diagnostische Aspekte neuropathischer Schmerzen

$13.00-13.30$

$13.30-14.00$

$14.00-14.30$

$14.30-15.00$

Update IASP Schmerzdiagnosen: Nozizeptiver, neuropathischer und noziplastischer Schmerz Quantitative sensorische Testung in der Diagnostik neuropathischer und nicht neuropathischer Schmerzen

Klinische und neurophysiologische Diagnostik neuropathischer Schmerzen bei Querschnittlähmung

Chronisch postoperativer Schmerz

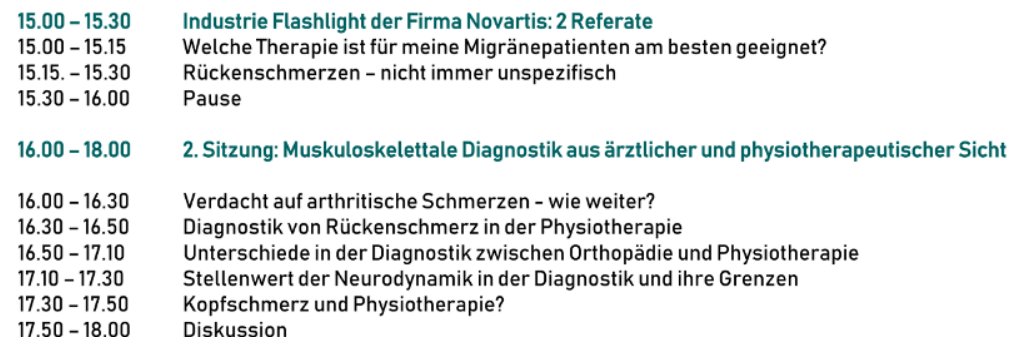
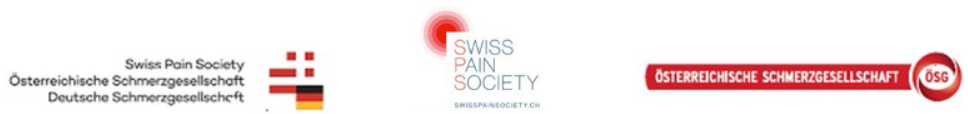

Achtung: Findet online statt!!!

\section{Samstag, 24. April 2021}

\begin{tabular}{|c|c|}
\hline $08.30-10.30$ & 1. Sitzung: Diagnostik psychischer Belastungsfaktoren \\
\hline $\begin{array}{l}08.30-08.50 \\
08.50-09.20\end{array}$ & $\begin{array}{l}\text { Schmerzpsychologische Diagnostik im interdisziplinären Team } \\
\text { Diagnostik und Therapie am ZSM aus psychiatrischer und psychologischer Sicht }\end{array}$ \\
\hline $\begin{array}{l}09.20-09.40 \\
09.40-10.00\end{array}$ & $\begin{array}{l}\text { Perceived Injustice als Therapieblocker } \\
\text { Die Entwicklung eines mehrdimensionalen Messinstruments für die IMST- was lernen wir } \\
\text { aus dem Prozess für die Therapie? }\end{array}$ \\
\hline $\begin{array}{l}10.00-10.20 \\
10.20-10.40\end{array}$ & $\begin{array}{l}\text { Interdisziplinarität } \\
\text { Pflegediagnose Schmerz - Bedeutung für die interprofessionelle Zusammenarbeit }\end{array}$ \\
\hline $10.40-11.10$ & Pause \\
\hline $\begin{array}{l}11.10-12.00 \\
11.10-11.30 \\
11.30-11.50 \\
11.50-12.00\end{array}$ & $\begin{array}{l}\text { 2. Sitzung: Ungewohnte aber wichtige Blickwinkel } \\
\text { Einbezug der spirituellen Dimension in die multimodale Schmerztherapie } \\
\text { Schmerz und Leid: eine philosophische Betrachtung } \\
\text { Diskussion }\end{array}$ \\
\hline $\begin{array}{l}12.00-12.30 \\
12.30-13.00\end{array}$ & $\begin{array}{l}\text { Industrie Flashlight Firma Elosan AG, Grabs } \\
\text { Elosan-Kabine zur Ganzkörper-Schmerztherapie } \\
\text { Lunch }\end{array}$ \\
\hline $13.00-15.00$ & $\begin{array}{l}\text { 3. Sitzung: Interventionelle Diagnostik in der Schmerzmedizin } \\
\text { Intrathekale Therapie }\end{array}$ \\
\hline $13.00-13.15$ & Testphase - Katheterverfahren versus single-shot: Referat: pro Katheter \\
\hline $13.15-13.30$ & Testphase - Katheterverfahren versus single-shot: Referat: pro single shot \\
\hline $13.30-13.45$ & Vorstellung des Algorithmus in Nottwil \\
\hline $13.45-13.50$ & Diskussion \\
\hline $13.50-14,30$ & Diagnostische Blockaden \\
\hline $13.50-14.10$ & Stellenwert und Durchführung diagnostischer zervikaler "medial branch"- Blockaden \\
\hline $14.10-14.25$ & Die diagnostische Blockade des peripheren Nerven war positiv - UND JETZT? \\
\hline $14.25-14.30$ & Diskussion \\
\hline $14.30-14.45$ & Gemeinsames Schlusswort Maurer, Mitrovic, Meissner, Ljutow \\
\hline
\end{tabular}

Vorsitz: U. Galli, Zürich /

A. Diezemann-Prössdorf, Mainz

B. Strock, Wien

S. Brockmüller, Nottwil /

J. Kaufmann, Nottwil

A. Diezemann-Prössdorf, Mainz

U. Kaiser, Dresden

A. Ott. St. Gallen

Prof. Dr. Nadja Nestler. Salzburg

Vorsitz: A. Ljutow, Nottwil

S. Peng-Keller, Zürich/K. Hasenfratz, Zürich

T. Eichinger, Zürich

Vorsitz: T. Reck, Nottwil, H. Likar, Klagenfurt

H. Likar, Klagenfurt

C. Höfing. Koblenz

C. Yetimoglu, Nottwil

K. Maurer, Zürich

J. Vera, Nottwil 


\section{Nachwuchsförderpreis Schmerz 2021}

\author{
Bewerbungsschluss: 31. Mai 2021
}

Der Nachwuchsförderpreis Schmerz wendet sich explizit an junge Wissenschaftlerinnen und Wissenschaftler an deutschen Forschungseinrichtungen, kurz vor oder kurz nach Promotionsabschluss, die sich für eine Forschungskarriere auf dem Gebiet der Schmerzforschung entschieden haben. Die bisherigen Forschungsaktivitäten sollten einen deutlichen Bezug zum Thema Schmerz aufweisen und die Grundlage für das neue Projektvorhaben bilden. Dieses sollte der patientenbezogenen Forschung zuzuordnen sein.

Ausgezeichnet wird die Nachwuchswissenschaftlerin oder der Nachwuchswissenschaftler mit dem eigenen innovativen Projektvorhaben, das einen wesentlichen Beitrag zur Therapie akuter und chronischer Schmerzen erwarten lässt. Das Ziel des Preises ist es, Nachwuchswissenschaftlerinnen und Nachwuchswissenschaftler mit ihren ausgezeichneten Arbeiten durch die Preisverleihung in den Fokus der Öffentlichkeit zu rücken und damit die Chancen auf weitere Förderung zu erhöhen.

Der Preis wird jährlich von der Deutschen Schmerzgesellschaft vergeben und dieses Jahr in Kooperation mit der Firma EVER Pharma GmbH ausgeschrieben und im Rahmen des Deutschen Schmerzkongresses der Deutschen Schmerzgesellschaft (20.-23. Oktober 2021 in Mannheim) verliehen.

Die drei besten Arbeiten werden mit 6000 Euro, 3000 Euro \& 1000 Euro prämiert. Über die Zuerkennung des Preises entscheidet eine Jury, die sich aus Hochschullehrern deutscher Universitäten zusammensetzt. Die Jury kann die Verleihung des Preises aussetzen, wenn keine preiswürdige Arbeit vorliegt. Sie kann auch Preise geteilt vergeben. Die Entscheidung der Jury ist für alle Beteiligten verbindlich und gerichtlich nicht anfechtbar. Die Bekanntgabe der Preisträger erfolgt in der deutschsprachigen Fachpresse. Die Preisträger werden schriftlich informiert.

Ausgezeichnet werden Projektvorhaben, die im Bereich der Schmerzforschung zukunftsweisend sind, das heißt zum Beispiel neue Methoden einsetzen oder etablierte Methoden auf neue Art und Weise kombinieren. Die Projekte sollten noch nicht anderweitig gefördert sein, können aber Spin-offs von bereits anderweitig geförderten Projekten sein. Eine zeitgleiche Bewerbung um den Nachwuchsförderpreis Schmerz mit einer Bewerbung für andere Preise ist nicht möglich.

Link zur Homepage:

https://www.schmerzgesellschaft.de/topnavi/forschung-und-foerderung/ forschungsfoerderung/nachwuchsfoerderpreis-schmerz

Bei der Bewerbung zusätzlich zu den im Bewerbungsformular anzugebende einzureichende Informationen/Unterlagen:

- Lebenslauf

- Verzeichnis der bisherigen Veröffentlichungen (falls schon vorhanden) und Anlage der Artikel (maximal 5)

- Beschreibung der Promotion und Darstellung, ob oder wann diese abgeschlossen ist (maximal 2 Seiten, Schrift Calibri 11, 1,5 zeilig)

- Beschreibung des Projektvorhabens (maximal 5 Seiten, Schrift Calibri 11, 1,5 zeilig)

- Forschungsaffiliation, kurzes Empfehlungsanschreiben der Doktormutter/des Doktorvaters, aus dem hervorgeht, ob das skizzierte und eingereichte Projektvorhaben realisierbar ist

- Erklärung darüber, dass neben der Bewerbung um den Nachwuchsförderpreis Schmerz zeitgleich keine Bewerbung

für andere Preise vorliegt

\section{Sponsor}

EVER PHARMA GmbH

Frau Eva Skarke

Oppelner Straße 5

82194 Gröbenzell

Tel.: +49 814242225233

E-Mail: eva.skarke@everphar-

ma.com

Web: www.everpharma.de
Weitere Informationen erhalten Sie unter:

Deutsche Schmerzgesellschaft e. V.

Alt-Moabit 101b

10559 Berlin

Tel.: +49 30394096 89-0

E-Mail: info@schmerzgesellschaft.de

Web: https://www.schmerzgesellschaft.de/

\section{Förderpreis für Schmerzforschung 2021}

\section{Ende der Bewerbungsfrist: 31. Mai 2021}

Zum 35. Mal zeichnet die Deutsche Schmerzgesellschaft e.V. in diesem Jahr wissenschaftlichen Nachwuchs mit dem von der Grünenthal GmbH gestifteten Förderpreis für Schmerzforschung aus. Mit dem Preis werden Arbeiten im Bereich der anwendungsbezogenen Forschung und der umgesetzten Grundlagenforschung gefördert, die einen wesentlichen Beitrag zur interdisziplinären praxisbezogenen Therapie akuter und chronischer Schmerzen geleistet haben. Der Preis richtet sich vorzugsweise an den wissenschaftlichen Nachwuchs aus den Fachgebieten Medizin, Psychologie und Naturwissenschaften.

Bewerbungen können für Projekte aus einer der beiden folgenden Kategorien eingereicht werden:

Kategorie 1: Klinische Forschung

für Studien an Patienten.

Kategorie 2: Grundlagenforschung

für experimentelle Arbeiten oder Arbeiten an Probanden.

Auf der Basis der Entscheidung der Jury werden in den bei- den Kategorien jeweils ein erster Preis mit einer Zuwendung von $7000 €$ und ein zweiter Preis mit einer Zuwendung von $3500 €$ vergeben. Die Entscheidung der Jury ist endgültig und erfolgt unter Ausschluss des Rechtsweges. Die Jury hat das Recht, von der Vergabe eines oder mehrerer Preise wegen mangelnder Preiswürdigkeit der eingereichten Arbeiten abzusehen. Ebenso kann die Jury jeden der Preise aufteilen. Die Preisträger werden schriftlich informiert. Die Verleihung der Preise und Bekanntgabe der Gewinner findet im Rahmen des Deutschen Schmerzkongresses vom 20.-23.10.2021 in Mannheim statt.

Eine Bewerbung ist ausschließlich in digitaler Form möglich. Die Arbeiten sind bis zum Ende der genannten Bewerbungsfrist (23.59 Uhr) als PDFDokument hochzuladen auf der Internetseite der Deutschen Schmerzgesellschaft:

https://www.schmerzgesellschaft.de/topnavi/forschung-und-foerderung/ forschungsfoerderung/foerderpreis-fuer-schmerzforschung 